Submitted to the Annals of Statistics

arXiv: arXiv: 1311.4104

\title{
SUPPLEMENT OF INTERMITTENT PROCESS ANALYSIS WITH SCATTERING MOMENTS
}

\author{
Appendix A: Proof of Theorem 2.1
}

Let us first prove (10). If $X$ is a Poisson process then $X \star \psi_{j}=2^{j} d X \star$ $\bar{\psi}_{j}$ where $\bar{\psi}(t)=\int_{0}^{t} \psi(u) d u$ has a support in $[-1 / 2,1 / 2]$, and $\bar{\psi}_{j}(t)=$ $2^{-j} \bar{\psi}\left(2^{-j} t\right)$. Since $d X(t)=\sum_{i} \delta\left(t-\tau_{i}\right)$ we get $X \star \psi_{j}(t)=2^{j} \sum_{i} \bar{\psi}_{j}\left(t-\tau_{i}\right)$. We write

$$
\left|X \star \psi_{j}(t)\right|=2^{j}\left|d X \star \bar{\psi}_{j}\right|(t)=2^{j}\left(d X \star\left|\bar{\psi}_{j}\right|(t)+e_{j}(t)\right) .
$$

The first term satisfies

$$
\mathbf{E}\left(d X \star\left|\bar{\psi}_{j}\right|\right)=\lambda\left\|\bar{\psi}_{j}\right\|_{1}=\lambda\|\bar{\psi}\|_{1} .
$$

Let us show that $\mathbf{E}\left(\left|e_{j}(t)\right|\right)=O\left(\lambda^{2} 2^{j}\right)$. Let $N_{j}(t)$ be the number of events counted by $X(t)$ in the interval $\left[t-2^{j}, t+2^{j}\right)$. We decompose

$\mathbf{E}\left(\left|e_{j}(t)\right|\right)=\mathbf{E}\left(\left|e_{j}(t)\right| / N_{j}(t) \leq 1\right) \operatorname{Prob}\left(N_{j}(t) \leq 1\right)+\mathbf{E}\left(\left|e_{j}(t)\right| / N_{j}(t)>1\right) \operatorname{Prob}\left(N_{j}(t)>1\right)$.

If $N_{j}(t) \leq 1$, since $\bar{\psi}_{j}$ has support $\left[-2^{j-1}, 2^{j-1}\right]$, it results that $e_{j}(t)=0$, and hence $\mathbf{E}\left(\left|e_{j}(t)\right| / N_{j}(t) \leq 1\right)=0$. Since

$$
\left|e_{j}(t)\right| \leq 2\left(d X \star\left|\bar{\psi}_{j}\right|(t)\right) \leq 2^{j+1}\|\bar{\psi}\|_{\infty} N_{j}(t)
$$

it follows that

$$
\mathbf{E}\left(\left|e_{j}(t)\right|\right) \leq 2\|\bar{\psi}\|_{\infty} 2^{j} \mathbf{E}\left(N_{j}(t) / N_{j}(t)>1\right) \operatorname{Prob}\left(N_{j}(t)>1\right) .
$$

Since $N_{j}(t)$ is a Poisson random variable of parameter $\lambda 2^{j}$, we verify that

$$
\mathbf{E}\left(N_{j}(t) / N_{j}(t)>1\right) \operatorname{Prob}\left(N_{j}(t)>1\right)=\lambda 2^{j}\left(1-e^{-\lambda 2^{j}}\right),
$$

which implies from (49) that

$$
\mathbf{E}\left(\left|e_{j}(t)\right|\right) \leq 2 \lambda\|\bar{\psi}\|_{\infty}\left(1-e^{-\lambda 2^{j}}\right)=O\left(\lambda^{2} 2^{j}\right),
$$

and, together with (48) and (47) proves (10).

Property (11) is proved by showing that $2^{-j / 2} X \star \psi_{j}(t)$ converges to a Gaussian random process at large scales $2^{j}$. The convergence of $2^{-j / 2} X \star$ 
$\psi_{j}(t)$ relies on the use of a central-limit theorem for real dependent random variables. The extension to the two-dimensional complex random variables is done by considering arbitrary linear combinations of its real and imaginary parts. The Cramer-Wold theorem proves that if $X_{j}=2^{-j / 2} X \star \psi_{j}(t)=$ $2^{-j / 2} \operatorname{Re}\left(X_{j}\right)+i 2^{-j / 2} \operatorname{Im}\left(X_{j}\right)$ satisfies

$$
\forall(\alpha, \beta) \in \mathbb{R}^{2} \quad, \quad \alpha \operatorname{Re}\left(X_{j}\right)+\beta \operatorname{Im}\left(X_{j}\right) \underset{j \rightarrow \infty}{\stackrel{l}{\longrightarrow}} \alpha A_{1}+\beta A_{2}
$$

then $X_{j} \underset{j \rightarrow \infty}{\stackrel{l}{\longrightarrow}} A_{1}+i A_{2}$. The random variables $A_{1}$ and $A_{2}$ are zero-mean Gaussian random variables if and only if $\alpha A_{1}+\beta A_{2}$ is a centered Gaussian random variable for all $(\alpha, \beta) \in \mathbb{R}^{2}$. But

$$
\alpha \operatorname{Re}\left(X_{j}\right)+\beta \operatorname{Im}\left(X_{j}\right)=X \star\left(\alpha \operatorname{Re}\left(\psi_{j}\right)+\beta \operatorname{Im}\left(\psi_{j}\right)\right)
$$

so the convergence of $X_{j}$ to a complex Gaussian variable will follow by showing that $2^{-j / 2} X \star \tilde{\psi}_{j} \rightarrow \mathcal{N}\left(0, \sigma^{2}\right)$ for any wavelet of the form $\tilde{\psi}_{j}=$ $\alpha \operatorname{Re}\left(\psi_{j}\right)+\beta \operatorname{Im}\left(\psi_{j}\right)$.

We thus concentrate in the real case, and we denote the real wavelet $\psi_{j}$ to simplify notations. Assuming $j>0$,

$$
X \star \psi_{j}(t)=2^{j} d X \star \bar{\psi}_{j}(t)=2^{j} \int_{-2^{j-1}}^{2^{j-1}} \bar{\psi}_{j}(u-t) d X(u)=\sum_{i=-2^{j-1}}^{2^{j-1}-1} S_{i, j},
$$

where $S_{i, j}=\int_{i}^{i+1} \bar{\psi}\left(2^{-j}(u-t)\right) d X(u)$ are a collection of zero-mean independent random variables. We apply the Berk central limit theorem [8], to this sum of independent random variables.

Theorem A.1 (Berk Central Limit). For any $j \in \mathbb{N}$, let $\left\{S_{i, j}\right\}_{i=1, \ldots, n_{j}}$ be a sequence of zero mean random variables such that for any $i \leq n_{j} S_{i, j}$ is independent of $S_{i+r, j}$ for $r \geq m_{j}$. If the following properties are satistied

(i) $\exists \delta>0, \lim _{j \rightarrow \infty} n_{j}^{-1} m_{j}^{2+2 / \delta}=0$

(ii) $\exists M>0, \forall i, j>0, \mathbf{E}\left(\left|S_{i, j}\right|^{2+\delta}\right) \leq M$

(iii) $\exists K>0, \forall i, j, l>k>0, \operatorname{Var}\left(\sum_{i=k+1}^{k+l} S_{i, j}\right) \leq l K$

(iv) $\lim _{j \rightarrow \infty} n_{j}^{-1} \operatorname{Var}\left(\sum_{i=1}^{n_{j}} S_{i, j}\right)=\sigma^{2}>0$

then

$$
n_{j}^{-1 / 2} \sum_{i=1}^{n_{j}} S_{i, j} \underset{j \rightarrow \infty}{\stackrel{l}{\longrightarrow}} \mathcal{N}\left(0, \sigma^{2}\right)
$$

imsart-aos ver. 2013/03/06 file: multifractals_aos6.tex date: October 22, 2014 
Let us now verify the hypothesis of this central limit theorem, with $m_{j}=$ $1, n_{j}=2^{j}$ and $\delta=1$. Since the variables $\left(S_{i, j}\right)_{i}$ are independent, hypothesis (i) is verified with $m_{j}=1$. Moreover, we verify that

$$
\mathbf{E}\left(\left|S_{i, j}\right|^{q}\right) \leq\|\psi\|_{\infty}^{q} \mathbf{E}\left(\left|N_{0}\right|^{q}\right)
$$

where $N_{0}$ is the number of jumps of $d X$ in an interval of length 1 . Since it follows a Poisson distribution of parameter $\lambda$, it has finite moments. It results that hypothesis (ii) is verified for $\delta=1$. Since the $S_{i, j}$ are independent, $\operatorname{Var}\left(\sum_{i=k+1}^{k+l} u_{i, j}\right) \leq\|\psi\|_{\infty}^{2} l \mathbf{E}\left(\left|N_{0}\right|^{2}\right)$, which verifies hypothesis (iii). Finally, since $d X$ is a white noise of power spectrum $\lambda$

$$
2^{-j} \sum_{|i| \leq 2^{j}} \mathbf{E}\left(\left|S_{i, j}\right|^{2}\right)=2^{j} \mathbf{E}\left(\left|d X \star \bar{\psi}_{j}\right|^{2}\right)=2^{j} \sigma^{2}(d X)\left\|\bar{\psi}_{j}\right\|^{2}=\lambda\|\bar{\psi}\|^{2} .
$$

It verifies the last hypothesis (iv). Applying (A.1) and the Cramer-Wald theorem proves that $2^{-j / 2} X \star \psi_{j}(t)$ converges to a complex Gaussian distribution of total variance $\lambda\|\bar{\psi}\|^{2}$. In order to control the limit of first order moments, we use the following lemma on uniform integrability of sequences of random variables:

Lemma A.2. ([14], thm 6.1-6.2) Let $\left\{X_{j}\right\} j \in \mathbb{N}$ be a sequence of random variables. If $X_{j} \stackrel{d}{\rightarrow} X_{\infty}$ and

$$
\sup _{j} \mathbf{E}\left(\left|X_{j}\right|^{1+\delta}\right)<\infty \text { for } \delta>0
$$

then

$$
\lim _{j \rightarrow \infty} \mathbf{E}\left(X_{j}\right)=\mathbf{E}\left(X_{\infty}\right)
$$

As a result, for any $\alpha \leq 2, \mathbf{E}\left(\left|2^{-j / 2} X \star \psi_{j}\right|^{\alpha}\right) \rightarrow \mathbf{E}\left(\left|Z_{1}+i Z_{2}\right|^{\alpha}\right)$, where $Z_{1}$ and $Z_{2}$ are Gaussian random variables with total variance $\lambda\|\bar{\psi}\|^{2}$. For $\alpha=1$, it results that there exists a constant $C$, depending only on the wavelet $\psi$, such that

$$
\lim _{j \rightarrow \infty} 2^{-j / 2} \mathbf{E}\left(\left|X \star \psi_{j}\right|\right)=\lambda^{1 / 2}\|\bar{\psi}\| C
$$

which proves (11).

The proof of (12) is very similar to the proof of (10). The key property is that ||$X \star \psi_{j_{1}}\left|\star \psi_{j_{2}}\right|$ only depends on values of $X$ over an interval of size $2^{j_{1}}+2^{j_{2}} \cdot$ ¿From (50), it results that

$\left|X \star \psi_{j_{1}}\right| \star \psi_{j_{2}}(t)=2^{j_{1}}\left|d X \star \bar{\psi}_{j_{1}}\right| \star \psi_{j_{2}}(t) \stackrel{d}{=} 2^{j_{1}}\left(d X \star\left(\left|\bar{\psi}_{j_{1}}\right| \star \psi_{j_{2}}\right)(t)+e_{j_{1}} \star \psi_{j_{2}}\right)$,

imsart-aos ver. 2013/03/06 file: multifractals_aos6.tex date: October 22, 2014 
with $\mathbf{E}\left(\left|e_{j_{1}} \star \psi_{j_{2}}\right|\right)=O\left(\lambda^{2} 2^{j_{1}}\right)$. As a consequence, (53) and (10) imply that

$$
\begin{array}{ll}
\mathbf{E}\left(|| X \star \psi_{j_{1}}\left|\star \psi_{j_{2}}(t)\right|\right) & =2^{j_{1}}\left(\mathbf{E}\left(\left|d X \star\left(\left|\bar{\psi}_{j_{1}}\right| \star \psi_{j_{2}}\right)\right|(t)\right)+O\left(\lambda^{2} 2^{j_{1}}\right)\right), \\
& =2^{j_{1}} \lambda\left\|\left(\left|\bar{\psi}_{j_{1}}\right| \star \psi_{j_{2}}\right)\right\|_{1}\left(1+O\left(\lambda 2^{j_{1}}\right)+O\left(\lambda 2^{j_{2}}\right)\right) .
\end{array}
$$

Using again (10), we conclude that

$$
\begin{aligned}
\tilde{S} X\left(j_{1}, j_{2}\right) & =\frac{\mathbf{E}\left(|| d X \star \bar{\psi}_{j_{1}}\left|\star \psi_{j_{2}}(t)\right|\right)}{\mathbf{E}\left(\left|d X \star \bar{\psi}_{j_{1}}\right|\right)} \\
& =\frac{\left\|\left(|\bar{\psi}| \star \psi_{j_{2}-j_{1}}\right)\right\|_{1}}{\|\bar{\psi}\|_{1}}\left(1+O\left(\lambda 2^{j_{1}}\right)+O\left(\lambda 2^{j_{2}}\right)\right)
\end{aligned}
$$

which proves (12). Finally, in order to prove (13), observe that $\left|X \star \psi_{j}\right|$ is a stationary process with lag $2^{j}$. As a result, by using the same Central Limit argument to prove (11), one can verify that $2^{j_{2} / 2}\left(\left|X \star \psi_{j_{1}}\right| \star \psi_{j_{2}}\right)$ converges in distribution towards a Gaussian distribution as $j_{2} \rightarrow \infty$, which yields a decay on the normalized second order scattering of the form $\tilde{S} d X\left(j_{1}, j_{2}\right) \simeq 2^{-j_{2} / 2}$ as $j_{2} \rightarrow \infty$.

\section{Appendix B: Proof of Theorem 3.2}

Proposition 3.1 proves in (16) that $\widetilde{S} X\left(j_{1}, j_{1}+j\right)=\mathbf{E}\left(\left|\widetilde{X} \star \psi_{j}\right|\right)$ and $\widetilde{X}(t)=|X \star \psi(t)| / \mathbf{E}(|X \star \psi|)$. We denote $\widetilde{S}_{2} X(j)=\mathbf{E}\left(\left|\widetilde{X} \star \psi_{j}\right|\right)$. Let $B(t)$ be a Brownian motion and $d B(t)$ be the Wiener measure. The two processes $X \star \psi(t)$ and $d^{H-1} d B \star \psi(t)$ are Gaussian stationary processes having same power spectrum so

$$
\{|X \star \psi(t)|\}_{t} \stackrel{d}{=}\left\{\left|d^{H-1} d B \star \psi(t)\right|\right\}_{t} \stackrel{d}{=}\left\{\left|d B \star d^{H-1} \psi(t)\right|\right\}_{t} .
$$

It results that

$$
\widetilde{S}_{2} X(j)=\frac{\mathbf{E}\left(|| d B \star d^{H-1} \psi\left|\star \psi_{j}\right|\right)}{\mathbf{E}(|X \star \psi|)}
$$

Since $\psi$ is $\mathbf{C}^{1}$, with a compact support and two vanishing moments, one can verify that $\left|d^{H-1} \psi(u)\right|=O\left(\left(1+|u|^{2}\right)^{-1}\right)$. It results that $\left|d B \star d^{H-1} \psi\right|$ is stationary process whose autocorrelation has some decay. As the scale $2^{j}$ increases, the second convolution with $\psi_{j}$ performs a progressively wider averaging. By applying a central-limit theorem for dependent random variables, the following lemma applied to $\varphi=d^{H-1} \psi$ proves that $2^{j / 1}\left|d B \star d^{H-1} \psi\right| \star \psi_{j}$ converges to a Gaussian processes and that its first moment converges to a constant when $j$ goes to $\infty$. The theorem result (19) stating that $2^{j / 2} \widetilde{S}_{2} X(j)$ converges to a constant results from (55).

imsart-aos ver. 2013/03/06 file: multifractals_aos6.tex date: October 22, 2014 
Lemma B.1. If $\varphi(u)=O\left(\left(1+|u|^{2}\right)^{-1}\right)$ then

$$
2^{j / 2}|d B \star \varphi| \star \psi_{j}(t) \underset{j \rightarrow \infty}{\stackrel{l}{\longrightarrow}} \mathcal{N}\left(0, \sigma^{2} \mathrm{Id}\right),
$$

with $\sigma^{2}=\|\psi\|_{2}^{2} \int R_{Y}(\tau) d \tau$ and

$$
\lim _{j \rightarrow \infty} \mathbf{E}\left(\left|2^{j / 2}\right| d B \star \varphi\left|\star \psi_{j}\right|\right)=\sigma \sqrt{\frac{\pi}{2}} .
$$

Appendix C: Proof of lemma B.1

Let $Y_{j}(t)=2^{j / 2}|d B \star \varphi| \star \psi_{j}(t)$. To prove that $\mathbf{E}\left(\left|Y_{j}\right|\right)$ converges to a constant, we shall prove that the distribution of $Y_{j}$ is asymptotically Gaussian:

$$
Y_{j}(t) \underset{j \rightarrow \infty}{\stackrel{l}{\longrightarrow}} A=A_{1}+i A_{2}
$$

where $A_{1}$ and $A_{2}$ are two zero-mean Gaussian distributions of total variance $\sigma_{1}^{2}+\sigma_{2}^{2}=\|\psi\|_{2}^{2} \int R_{|d B \star \varphi|}(\tau) d \tau$, which is the first result of Lemma B.1. We shall also prove that

$$
\lim _{j \rightarrow \infty} \mathbf{E}\left(\left|Y_{j}\right|^{2}\right)=\mathbf{E}\left(|A|^{2}\right)
$$

Using again lemma (A.2), we conclude that

$$
\lim _{j \rightarrow \infty} \mathbf{E}\left(\left|Y_{j}\right|\right)=\mathbf{E}(|A|)>0 .
$$

and hence finish the proof of Lemma B.1.

For that purpose, we follow the same strategy as in the proof of (11), applied to the process $Y_{j}=2^{j / 2}|d B \star \varphi| \star \bar{\psi}_{j}(t)$, where $\bar{\psi}_{j}$ is any linear combination of real and imaginary parts of $\psi_{j}$.

Let us write $\varphi_{\Delta}=\varphi \mathbf{1}_{[-\Delta / 2, \Delta / 2]}$. We shall limit $\phi$ to a compact support by defining $\left\{\Delta_{j}\right\}_{j \geq 0}$ with $\lim _{j \rightarrow \infty} \Delta_{j}=\infty$ and decompose

$$
|d B \star \varphi(t)|=\left|d B \star \varphi_{\Delta_{j}}+d B \star\left(\varphi-\varphi_{\Delta_{j}}\right)\right| .
$$

As a result

$$
|d B \star \varphi(t)|=\left|d B \star \varphi_{\Delta_{j}}\right|+Z_{j}(t)
$$

with $\mathbf{E}\left(\left|Z_{j}\right|\right) \leq \mathbf{E}\left(\left|d B \star\left(\varphi-\varphi_{\Delta_{j}}\right)\right|\right)$. Since $d B$ is the Wiener measure, if $\theta \in \mathbf{L}^{2}(\mathbb{R})$ then

$$
\mathbf{E}(|d B \star \theta|) \leq \mathbf{E}\left(|d B \star \theta|^{2}\right)^{1 / 2}=\|\theta\|_{2}
$$

imsart-aos ver. 2013/03/06 file: multifractals_aos6.tex date: October 22, 2014 
so $\mathbf{E}\left(\left|Z_{j}\right|\right) \leq\left\|\varphi-\varphi_{\Delta_{j}}\right\|_{2}$. It results that

$$
|d B \star \varphi| \star \bar{\psi}_{j}(t)=\left|d B \star \varphi_{\Delta_{j}}\right| \star \bar{\psi}_{j}(t)+Z_{j} \star \bar{\psi}_{j}(t),
$$

and

$$
\mathbf{E}\left(\left|Z_{j} \star \bar{\psi}_{j}\right|\right) \leq \mathbf{E}\left(\left|Z_{j}\right|\right)\left\|\bar{\psi}_{j}\right\|_{1} \leq\left\|\varphi-\varphi_{\Delta_{j}}\right\|_{2}\|\bar{\psi}\|_{1} .
$$

Since $\lim _{j \rightarrow \infty} \Delta_{j}=\infty, \lim _{j \rightarrow \infty}\left\|\varphi-\varphi_{\Delta_{j}}\right\|_{2}=0$ so $Z_{j} \star \bar{\psi}_{j}$ converges to 0 in probability when $j$ increases. So the limits of $|d B \star \varphi| \star \bar{\psi}_{j}(t)$ and $\mid d B \star$ $\varphi_{\Delta_{j}} \mid \star \bar{\psi}_{j}(t)$ are equal.

We now prove (56) by applying Berk central limit Theorem A.1, to show that $\bar{Y}_{j}=\left|d B \star \varphi_{\Delta_{j}}\right| \star \bar{\psi}_{j}(t)$ converges to a normal distribution. Since $\mid d B \star$ $\varphi_{\Delta_{j}} \mid \star \bar{\psi}_{j}(t)$ is stationary, its distribution can be evaluated at $t=0$

$$
\left|d B \star \varphi_{\Delta_{j}}\right| \star \bar{\psi}_{j}(0)=\int\left|d B \star \varphi_{\Delta_{j}}\right|(u) \bar{\psi}_{j}(-u) d u .
$$

The central-limit theorem is applied by dividing this integral into disjoint integrals

$$
S_{i, j}=2^{j} \int_{2^{j} b_{i, j}}^{2^{j} b_{i+1, j}}\left|d B \star \varphi_{\Delta_{j}}(u)\right| \bar{\psi}_{j}(-u) d u,
$$

where for each $j \in \mathbb{Z},\left\{b_{i, j}\right\}_{1 \leq i \leq n_{j}}$ is an increasing sequence of points in $\mathbb{R} \cup\{ \pm \infty\}$ such that

$$
\forall i, \quad \int_{b_{i, j}}^{b_{i+1, j}}|\bar{\psi}(-u)| d u=2^{-j}\|\bar{\psi}\|_{1} .
$$

Since $\bar{\psi}$ is $\mathbf{C}^{\mathbf{1}}$ and bounded, we verify that $n_{j} \simeq 2^{j}$. Summing these random variables gives

$$
2^{-j / 2} \sum_{i=1}^{n_{j}} S_{i, j}=2^{j / 2}\left|d B \star \varphi_{\Delta_{j}}\right| \star \bar{\psi}_{j}(0) .
$$

We now show that the $S_{i, j}$ satisfy the hypothesis of the Beck central-limit theorem so that we can apply the convergence result (52) which implies (56).

Let us first prove that $S_{i, j}$ is independent of $S_{i+r, j}$ for $r \geq m_{j}$ which satisfies (i). Since $\bar{\psi}$ is bounded, it results that $\inf _{i, j} 2^{j}\left|b_{i, j}-b_{i+1, j}\right|=\eta>0$. Since $\varphi_{\Delta_{j}}$ has a support of size $\Delta_{j}$ and $d B$ is a Wiener Noise, it follows that $\left|d B \star \varphi_{\Delta_{j}}\right|(u)$ is independent of $\left|d B \star \varphi_{\Delta_{j}}\right|\left(u^{\prime}\right)$ for $\left|u-u^{\prime}\right|>\Delta_{j}$ and hence that $S_{i, j}$ is independent of $S_{i+r, j}$ for $r \geq m_{j}=\Delta_{j} / \eta$.

imsart-aos ver. 2013/03/06 file: multifractals_aos6.tex date: October 22, 2014 
To verify (i) let us set $\delta=1$. Since $n_{j} \simeq 2^{j}$, if we choose $\Delta_{j}=2^{j / 5}$ then

$$
\lim _{j \rightarrow \infty} \frac{m_{j}^{4}}{n_{j}} \leq \eta^{-4} \lim _{j \rightarrow \infty} 2^{j(4 / 5-1)}=0 .
$$

We now verify condition (ii) with $\delta=1$. Since $\bar{\psi}_{j}(u)$ has a zero integral, one can replace $\left|d X \star \varphi_{\Delta_{j}}(u)\right|$ by $Q_{j}(u)=\left|d X \star \varphi_{\Delta_{j}}\right|(u)-\mathbf{E}\left(\left|d X \star \varphi_{\Delta_{j}}\right|\right)$ in the definition (63) of $S_{i, j}$. It results that

$$
\begin{aligned}
\mathbf{E}\left(\left|S_{i, j}\right|^{3}\right) & \leq \iiint \mathbf{E}\left(Q_{j}(u) Q_{j}\left(u^{\prime}\right) Q_{j}\left(u^{\prime \prime}\right)\right) 2^{3 j}\left|\bar{\psi}_{j}(-u)\right|\left|\bar{\psi}_{j}\left(-u^{\prime}\right)\right|\left|\bar{\psi}_{j}\left(-u^{\prime \prime}\right)\right| d u d u^{\prime} d u^{\prime \prime} \\
& \leq \mathbf{E}\left(\left|d B \star \varphi_{\Delta_{j}}\right|^{3}\right)\|\bar{\psi}\|_{1}^{3}=2^{5 / 2} \pi^{-1 / 2}\left\|\varphi_{\Delta_{j}}\right\|_{2}^{3}\|\bar{\psi}\|_{1}^{3} \leq 2^{5 / 2} \pi^{-1 / 2}\|\varphi\|_{2}^{3}\|\bar{\psi}\|_{1}^{3} .
\end{aligned}
$$

Let us now verify condition (iii). The sum $A_{k, l, j}=\sum_{i=k}^{k+l} S_{i, j}$ is by definition

$$
A_{k, l, j}=2^{j} \int_{2^{j} b_{k, j}}^{2^{j} b_{k+l, j}}\left|d B \star \varphi_{\Delta_{j}}(u)\right| \bar{\psi}_{j}(-u) d u=\int_{\mathbb{R}}\left|d B \star \varphi_{\Delta_{j}}(u)\right| h_{k, l, j}(u) d u
$$

with $h_{k, l, j}(u)=2^{j} \bar{\psi}_{j}(-u) 1_{\left[2^{j} b_{k, j}, 2^{j} b_{k+l, j}\right]}(u)$. It results that

$$
\operatorname{Var}\left(A_{k, l, j}\right) \leq\left\|R_{\left|d B \star \varphi_{\Delta_{j}}\right|}\right\|_{1}\left\|h_{k, l, j}\right\|_{2}^{2} .
$$

But, with a change of variable and applying (64) we get

$$
\left\|h_{k, l, j}\right\|_{2}^{2}=\int_{2^{j} b_{k, j}}^{2^{j} b_{k+l, j}}\left|\bar{\psi}\left(2^{-j} u\right)\right|^{2} d u \leq\|\bar{\psi}\|_{\infty} \int_{b_{k, j}}^{b_{k+l, j}} 2^{j}|\bar{\psi}(u)| d u \leq\|\bar{\psi}\|_{\infty}\|\bar{\psi}\|_{1} l .
$$

We are now going to bound $\left\|R_{\left|d B \star \varphi_{\Delta_{j}}\right|}\right\|_{1}$ by using the decay $\varphi(u)=$ $O\left(\left(1+|u|^{-2}\right)\right)$.

$$
R_{\left|d B \star \varphi_{\Delta_{j}}\right|}(\Delta)=\mathbf{E}\left(\left|d B \star \varphi_{\Delta_{j}}(\Delta)\right|\left|d B \star \varphi_{\Delta_{j}}(0)\right|\right)-\mathbf{E}\left(\left|d B \star \varphi_{\Delta_{j}}\right|\right)^{2} .
$$

If $|\Delta|>\left|\Delta_{j}\right|$ then since the support of $\varphi_{\Delta_{j}}(u)$ and $\varphi_{\Delta_{j}}(u-\Delta)$ does not overlap, $\left|d B \star \varphi_{\Delta_{j}}(\Delta)\right|$ and $\left|d B \star \varphi_{\Delta_{j}}(0)\right|$ are independent random variables so $R_{\left|d B \star \varphi_{\Delta_{j} \mid}\right|}(\Delta)=0$. Otherwise, we decompose

$$
\left|d B \star \varphi_{\Delta_{j}}(u)\right|=\mid d B \star \varphi_{\Delta}(u)+d B \star\left(\varphi_{\Delta_{j}}-\varphi_{\Delta}(u) \mid .\right.
$$

Since $\left|d B \star \varphi_{\Delta}(0)\right|$ and $\left|d B \star \varphi_{\Delta}(\Delta)\right|$ are independent random variables,

$$
\begin{aligned}
& \begin{aligned}
\left|R_{\left|d B \star \varphi_{\Delta_{j}}\right|}(\Delta)\right| \leq & \left|\mathbf{E}\left(\left|d B \star \varphi_{\Delta}\right|\right)^{2}-\mathbf{E}\left(\left|d B \star \varphi_{\Delta_{j}}\right|\right)^{2}\right|+ \\
& +2 \mathbf{E}\left(\left|d B \star \varphi_{\Delta}\right|\right) \mathbf{E}\left(\left|d B \star\left(\varphi_{\Delta_{j}}-\varphi_{\Delta}\right)\right|\right)+\mathbf{E}\left(\left|d B \star\left(\varphi_{\Delta_{j}}-\varphi_{\Delta}\right)\right|\right)^{2} .
\end{aligned} \\
& \text { imsart-aos ver. 2013/03/06 file: multifractals_aos6.tex date: October 22, } 2014
\end{aligned}
$$


Since $\mathbf{E}(|d B \star \theta|) \leq \mathbf{E}\left(|d B \star \theta|^{2}\right)^{1 / 2} \leq\|\theta\|_{2}$, by applying this to $\theta=\varphi_{\Delta}$ and $\theta=\varphi_{\Delta_{j}}-\varphi_{\Delta}$ one can verify that

$$
\left|R_{\left|d B \star \varphi_{\Delta_{j}}\right|}(\Delta)\right| \leq 6\|\varphi\|_{2}\left\|\varphi-\varphi_{\Delta}\right\|_{2} .
$$

Since $\varphi(u)=O\left((1+|u|)^{-2}\right)$ it results that $\left\|\varphi-\varphi_{\Delta}\right\|_{2}=O\left((1+|\Delta|)^{-3 / 2}\right)$ so $\left\|R_{\left|d B \star \varphi_{\Delta_{j}}\right|}\right\|_{1}$ is bounded independently of $j$. Inserting this in (67) proves the theorem hypothesis (iii).

Let us now verify the hypothesis (iv). It results from (65) that

$$
2^{-j} \operatorname{Var}\left(\sum_{i} S_{i, j}\right)=2^{j} \operatorname{Var}\left(\left|d X \star \varphi_{\Delta_{j}}\right| \star \bar{\psi}_{j}\right)=2^{j} \int \widehat{R}_{\mid d X \star \varphi_{\Delta_{j}}}(\omega)\left|\widehat{\bar{\psi}}\left(2^{j} \omega\right)\right|^{2} d \omega
$$

We proved (68) that $R_{\left|d B \star \varphi_{\Delta_{j}}\right|} \in \mathbf{L}^{1}$ but the same inequality is valid for $R_{\left|d B \star \varphi_{\Delta}\right|}$ which proves that it is also in $\mathbf{L}^{1}$. It results that $\widehat{R}_{|d X \star \varphi|}$ is continuous. Since $\varphi_{\Delta_{j}}$ converges to $\varphi$ in $\mathbf{L}^{2} \cap \mathbf{L}^{1}$ as $j \rightarrow \infty, \widehat{R}_{\left|d X \star \varphi^{j}\right|}(0)$ converges to $\widehat{R}_{|d X \star \varphi|}(0)$. Since $2^{j}\left|\widehat{\bar{\psi}}\left(2^{j} \omega\right)\right|^{2}$ converges to $\|\bar{\psi}\|_{2}^{2} \delta(\omega)$ when $j$ goes to $\infty$

$$
\lim _{j \rightarrow \infty} 2^{-j} \operatorname{Var}\left(\sum_{i} S_{i, j}\right)=\widehat{R}_{|d X \star \varphi|}(0)\|\bar{\psi}\|_{2}^{2}=\bar{\sigma}^{2},
$$

which proves condition (iv).

We can thus apply Theorem A.1 which proves that $2^{j / 2}|d B \star \varphi| \star \bar{\psi}_{j}(t)$ converges in distribution to $\mathcal{N}\left(0 \bar{\sigma}^{2}\right)$ and hence (56). Finally, by following the same reasoning used in Theorem 2.1, we apply Lemma A.2 to conclude that $\lim _{j \rightarrow \infty} 2^{j / 2} \mathbf{E}\left(|| d B \star \varphi\left|\star \psi_{j}\right|\right)=C>0$, which proves $(57) \square$.

Appendix D: Various results on the MRM measure

LEMmA D.1. [6] The process $\omega_{l}^{T}(t)$ used for the construction of the MRM $d M$ is an infinitely-divisible process whose two-points characteristic function reads:

$$
\mathbf{E}\left(e^{p_{1} \omega_{l}^{T}\left(t_{1}\right)+p_{2} \omega_{l}^{T}\left(t_{2}\right)}\right)=e^{\left[F\left(p_{1}\right)+F\left(p_{2}\right)\right] \rho_{l}^{T}(0)+\left[F\left(p_{1}+p_{2}\right)-F\left(p_{1}\right)-F\left(p_{2}\right)\right] \rho_{l}^{T}\left(t_{2}-t_{1}\right)}
$$

where $F(-i p)$ is the cumulant generating function characterizing the infinitely divisible law as provided by the Levy-Khintchine formula where the drift term is chosen such that $F(1)=0$, and where the function $\rho_{l}^{T}(\tau)$ is defined by:

$$
\rho_{l}^{T}(\tau)=\left\{\begin{aligned}
\ln (T / l)+1-|\tau| / l, & \text { if }|\tau| \leq l \\
\ln (T /|\tau|), & \text { if } l \leq|\tau|<T, \\
0, & \text { otherwise }
\end{aligned}\right.
$$

imsart-aos ver. 2013/03/06 file: multifractals_aos6.tex date: October 22, 2014 
Moreover, the function $\zeta(p)$ which satisfies (26) (with $X=d M$ where $d M$ is the associated $M R M)$ is given by $\zeta(p)=p-F(p)$.

The next Lemma uses an alternative MRM measure considered in [6] defined by

$$
d \tilde{M}(t)=\lim _{l \rightarrow 0} e^{\tilde{\omega}_{l}^{T}(t)} d t
$$

where $\tilde{\omega}_{l}^{T}$ is defined exactly as the process $\omega_{l}^{T}$ but only differs by its $\rho$ function which is replaced by $\tilde{\rho}_{l}^{T}(\tau)=\rho_{l}^{T}(\tau)+\frac{\tau}{T}-1$, for $\tau \leq T$. One can show that $\tilde{\omega}_{l}^{T}$ is linked with $\omega_{l}^{T}$ by the following cascade property:

$$
\forall l \leq a \leq T, \omega_{l}^{T}(u) \stackrel{a . s .}{=} \tilde{\omega}_{l}^{a}(u)+\omega_{a}^{T}(u)
$$

where $\tilde{\omega}_{l}^{a}$ and $\omega_{a}^{T}$ are independent copies of the processes defined previously. Moreover, $\tilde{\omega}_{l}^{T}$ satisfies both (31) and (31).

LEMMA D.2. Let $\omega_{l}^{T}$ the infinitely divisible process associated with the $M R M d M$ and $\psi$ be a wavelet of support in $[0,1]$ such that $\|\psi\|_{\infty}<\infty$. For all $\alpha$ such that $0<\alpha<1$, one has:

$$
\forall l<2^{j},\left(\psi_{j} \star e^{\omega_{l}^{T}}\right)(t)=e^{\omega_{2 j \alpha}^{T}(t)}\left(\psi_{j} \star e^{\tilde{\omega}_{l}^{2 \alpha}}\right)+\eta_{l, j}(t),
$$

where the process $\eta_{l, j}(t)$ has a limit process $\lim _{l \rightarrow 0} \eta_{l, j}(t)=\eta_{j}(t)$ which satisfies, in the limit $j \rightarrow-\infty$,

$$
\mathbf{E}\left(\left|\eta_{j}(t)\right|\right)=O\left(2^{j \frac{1-\alpha(1+F(2))}{2}}\right) .
$$

Proof: Without loss of generality we fix $t=0$. Let us consider $0<\alpha<1$ and $l$ and $j$ small enough and such that $l<2^{j}<2^{j \alpha}<T$. Let us first remark that, for $u<2^{j \alpha}$, one has from (69):

$$
\mathbf{E}\left(e^{p\left(\omega_{2 j \alpha}^{T}(u)+\omega_{2 j \alpha}^{T}(0)\right)}\right)=2^{-j \alpha F(2 p)} T^{F(2 p)} e^{F(2 p)\left(1-u 2^{-j \alpha}\right)}
$$

where $F(p)=\varphi(-i p)=p-\zeta(p)$. Hence, we have:

$\mathbf{E}\left(e^{2 \omega_{2^{j \alpha}}^{T}(u)}\right)=2^{-j \alpha F(2)} T^{F(2)} e^{F(2)}, \mathbf{E}\left(e^{\omega_{2^{j \alpha}}^{T}(u)+\omega_{2}^{T}{ }^{j \alpha}(0)}\right)=2^{-j \alpha F(2)} T^{F(2)} e^{F(2)\left(1-u 2^{-j \alpha}\right)}$.

One defines $\eta_{l, j}$ as:

$$
\eta_{l, j}(0)=2^{-j} \int \psi\left(u 2^{-j}\right)\left(e^{\omega_{l}^{T}(u)}-e^{\tilde{\omega}_{l}^{2 j \alpha}(u)+\omega_{2^{j \alpha}}^{T}(0)}\right) d u
$$

imsart-aos ver. 2013/03/06 file: multifractals_aos6.tex date: October 22, 2014 
Using dominated convergence, $(71), \mathbf{E}\left(e^{\tilde{\omega}_{l}^{2 \alpha}}\right)=1$ and the fact that $\psi$ is a bounded function of support $[0,1]$ one has:

$$
\begin{aligned}
\mathbf{E}\left(\lim _{l \rightarrow 0}\left|\eta_{l, j}\right|\right) & =\lim _{l \rightarrow 0} \mathbf{E}\left(\left|\eta_{l, j}\right|\right) \\
& \leq\|\psi\|_{\infty} 2^{-j} \int_{0}^{2^{j}} \sqrt{\mathbf{E}\left[\left(e^{\omega_{2 j \alpha}^{T}(u)}-e^{\omega_{2^{j \alpha}}^{T}(0)}\right)^{2}\right]} d u \\
& =\|\psi\|_{\infty} 2^{-j} \int_{0}^{2^{j}} \sqrt{\mathbf{E}\left(e^{2 \omega_{2^{j \alpha}}^{T}(0)}+e^{2 \omega_{2^{j \alpha}}^{T}(u)}-2 e^{\omega_{2^{j \alpha}}^{T}(u)+\omega_{2^{j \alpha}}^{T}(0)}\right)} d u \\
& =\sqrt{2}\|\psi\|_{\infty} 2^{-\frac{j \alpha F(2)}{2}} T^{\frac{F(2)}{2}} e^{\frac{F(2)}{2}} \int_{0}^{1}\left(1-e^{-F(2) u 2^{j(1-\alpha)}}\right)^{\frac{1}{2}} d u \\
& =O\left(2^{j \frac{1-\alpha(1+F(2))}{2}}\right) \square .
\end{aligned}
$$

Appendix E: Proof of Theorem 4.2

E.1. Proof of (i). We first prove that for all $t \in\left[0,2^{L}-1\right]$ and all $j<L$

$$
\frac{d M \star \psi_{j}}{\mathbf{E}(|d M \star \psi|)} \stackrel{\text { law }}{=} e^{\Omega_{2^{j-L}}} \xi\left(t 2^{-j}\right),
$$

where $\Omega_{s}$ is the random variable defined in $(32)$ and $\xi_{1}(t)$ is a normalized 1-dependent, stationary random process independent of $\Omega_{2^{j-L}}$ defined as:

$$
\xi(t)=K^{-1} \epsilon_{1}(t)
$$

where $\epsilon_{1}(t)=\lim _{l \rightarrow 0} \int \psi(u-t) e^{\omega_{l}^{1}(u)} d u$ and $K=\mathbf{E}\left(\left|\epsilon_{1}(t)\right|\right)$.

Let $j<L$ and $d M_{l}=e^{\omega_{l}^{2}}(t) d t$. From (31), one has:

$$
d M_{l} \star \psi_{j} \stackrel{\text { law }}{=} 2^{-j} \int e^{\omega_{2 j-L_{l}}^{2^{j}}\left(u 2^{j-L}\right)} \psi\left(2^{-j}(t-u)\right) d u,
$$

and thus, by setting $s=2^{j-L}$, from (32) and (31):

$$
d M_{l} \star \psi_{j} \stackrel{l a w}{=} 2^{-j} e^{\Omega_{s}} \int \psi\left(2^{-j}(t-u)\right) e^{\omega_{l}^{j}(u)} d u \stackrel{\text { law }}{=} e^{\Omega_{s}} \int \psi\left(2^{-j} t-u\right) e^{\omega_{l 2^{-j}}(u)} d u .
$$

Taking the limit $l \rightarrow 0$ for a fixed $j$ in the last equation shows that

$$
d M \star \psi_{j} \stackrel{\text { law }}{=} e^{\Omega_{2 j-L}} \epsilon_{1}\left(t 2^{-j}\right),
$$

with

$$
\epsilon_{T}(t)=\lim _{l \rightarrow 0} \int \psi(u-t) e^{\omega_{l}^{T}(u)} d u
$$

imsart-aos ver. 2013/03/06 file: multifractals_aos6.tex date: October 22, 2014 
Normalizing $\epsilon_{1}(t)$ by $E(|d M \star \psi|)$ proves $(76)$.

Since $\psi$ has a compact support of size 1 , the process $\epsilon_{1}(t)$ (and therefore the process $\left.\xi_{1}(t)\right)$ is a 1-dependent process, i.e., $\forall \tau>1, \epsilon_{1}(t+\tau)$ is independent from $\left\{\epsilon_{1}\left(t^{\prime}\right)\right\}_{t^{\prime} \leq t}$. Equation (33) is a direct consequence of (76) and of the fact that $E\left(e^{\Omega_{s}}\right)=1$.

E.2. Proof of (ii). As for the first order, using first (31) and then (32) with $s=2^{j_{2}-L}$ we obtain :

$$
\begin{aligned}
& \left|\psi_{j_{2}} \star\right| \psi_{j_{1}} \star d M_{l}||(t) \stackrel{\text { law }}{=}\left|\psi_{j_{2}} \star\right| \psi_{j_{1}} \star d M_{l}||(0) \\
& \stackrel{\text { law }}{=} 2^{-j_{2}} e^{\Omega_{2} j_{2}-L}\left|\int \psi\left(-u 2^{-j_{2}}\right) 2^{-j_{1}}\right| \int \psi\left(\frac{u-v}{2^{j_{1}}}\right) e^{\omega_{l}^{j_{2}}(v)} d v|d u| \text {. }
\end{aligned}
$$

Making the changes of variables $u^{\prime}=u 2^{-j_{2}}$ and $v^{\prime}=v 2^{-j_{1}}$ and using (31), leads to

$\left|\psi_{j_{2}} \star\right| \psi_{j_{1}} \star d M_{l}||(t) \stackrel{l a w}{=} e^{\Omega_{2} j_{2}-L}\left|\int \psi(-u)\right| \int \psi\left(2^{j_{2}-j_{1}} u-v\right) e^{\omega_{2^{2} j_{1}}^{j_{2}-j_{1}}(v)} d v|d u|$.

Since $j_{2}$ is fixed, with no loss of generality, in the following we can set $j_{2}=0$.

Using (31), one gets

$$
|\psi \star| \psi_{j_{1}} \star d M_{l}(0)|| \stackrel{\text { law }}{=} e^{\Omega_{2}-L}\left|\int \psi(-u)\right| \psi_{j_{1}} \star e^{\omega_{l}^{1}}(u)|d u| .
$$

We now use the Lemma D.2 proved in Appendix D with $\alpha=\frac{1-2 \nu}{1+F(2)}$ $(\nu<1 / 2)$. We get :

$$
\mathbf{E}\left(\left|\eta_{j_{1}}\right|\right)=O\left(2^{j_{1} \nu}\right)
$$

and

$$
\begin{aligned}
\psi_{j_{1}} \star e^{\omega_{l}^{1}}(u) & =2^{-j_{1}} e^{\omega_{2 j_{1} \alpha}^{1}(u)} \int \psi\left(\frac{u-v}{2^{j_{1}}}\right) e^{\tilde{\omega}_{l}^{2_{1}^{j_{1} \alpha}}(v)} d v+\eta_{j_{1}, l}(u) \\
& \stackrel{\operatorname{law}}{=} e^{\omega_{2^{j_{1} \alpha}}^{1}(u)} \int \psi\left(u 2^{-j_{1}}-v\right) e^{\tilde{\omega}_{l 2^{-j_{1} \alpha}}^{1}\left(v 2^{j_{1}(1-\alpha)}\right)} d v+\eta_{j_{1}, l}(u) \\
& \stackrel{\operatorname{law}}{=} e^{\omega_{2^{j_{1} \alpha}}^{1}(u)} \int \psi\left(u 2^{-j_{1}}-v\right) e^{\tilde{\omega}_{l 2^{-j_{1}}}^{j_{1}(\alpha-1)}(v)} d v+\eta_{j_{1}, l}(u) \\
& \underset{l \rightarrow 0}{\rightarrow} e^{\omega_{2^{j_{1} \alpha}}^{1}(u)} \tilde{\epsilon}_{2^{j_{1}(\alpha-1)}}\left(2^{-j_{1}} u\right)+\eta_{j_{1}}(u)
\end{aligned}
$$

where we used property (31) for $\tilde{\omega}_{l}^{T}$ and we defined the $T$-dependent noise:

$$
\tilde{\epsilon}_{T}(t)=\lim _{l \rightarrow 0} \int \psi(t-v) e^{\tilde{\omega}_{l 2}^{T}-j_{1}}(v) d v
$$

imsart-aos ver. 2013/03/06 file: multifractals_aos6.tex date: October 22, 2014 
If follows that :

$\lim _{l \rightarrow 0} \psi \star\left|\psi_{j_{1}} \star d M_{l}(0)\right| \stackrel{l a w}{=} e^{\Omega_{2}-L} \int \psi(-u) e^{\omega_{2}^{1 j_{1} \alpha}(u)}\left|\tilde{\epsilon}_{2^{j_{1}(\alpha-1)}}\left(2^{-j_{1}} u\right)\right| d u+\int \psi(-u) \tilde{\eta}_{j_{1}}(u) d u$,

where

$$
\tilde{\eta}_{j_{1}}(u)=\left|e^{\omega_{2^{j_{1} \alpha}}^{1}(u)} \tilde{\epsilon}_{2^{j_{1}(\alpha-1)}}\left(2^{-j_{1}} u\right)+\eta_{j_{1}}(u)\right|-\left|\tilde{\epsilon}_{2^{j_{1}(\alpha-1)}}\left(2^{-j_{1}} u\right)\right|
$$

Along the same line as in ref $[32,6]$, it is easy to prove that in the limit $T \rightarrow \infty$ :

$$
\mathbf{E}\left(\left|\tilde{\epsilon}_{T}(t)\right|^{q}\right) \simeq \widetilde{K}_{q} T^{q-\zeta(q)}
$$

where $\tilde{K}_{q}$ does not depend on $T$ (thanks to the stationarity of $\tilde{\epsilon}_{T}(t)$, it does not depend on $t$ either). Since $\zeta(1)=1$, let $\tilde{K}_{1}=\tilde{K}=\mathbf{E}\left(\left|\tilde{\epsilon}_{T}(t)\right|\right)$ and let us define the centered process: $\bar{\epsilon}_{T}(t)=\left|\tilde{\epsilon}_{T}(t)\right|-\tilde{K}$. Let us remark that, when $T \rightarrow \infty$

$$
\mathbf{E}\left(\bar{\epsilon}_{T}^{2}\right) \simeq \mathbf{E}\left(\tilde{\epsilon}_{T}^{2}\right) \simeq T^{2-\zeta(2)}
$$

Thus we can write

$$
\lim _{l \rightarrow 0} \psi \star\left|\psi_{j_{1}} \star d M_{l}\right|(0) \stackrel{\text { law }}{=} e^{\Omega_{2}-L}(I+I I+I I I),
$$

where

$$
\begin{aligned}
I & =\tilde{K} \int \psi(-u) e^{\omega_{2^{j_{1} \alpha}}^{1}(u)} d u, \\
I I & =\int \psi(-u) e^{\omega_{2^{j_{1} \alpha}}^{1}} \bar{\epsilon}_{2^{j_{1}(\alpha-1)}}\left(2^{-j_{1}} u\right) d u, \\
I I I & =\int \psi(-u) \tilde{\eta}_{j_{1}}(u) d u .
\end{aligned}
$$

Since $\int \psi(u) e^{\omega_{2 j_{1} \alpha}^{1}} d u$ converges in law, when $j_{1} \rightarrow-\infty$, towards $\epsilon_{1}(t)$, (where $\epsilon_{1}(t)$ is an independent copy of the process defined in (79)), we have, in the limit $j_{1} \rightarrow-\infty$ :

$$
\mathbf{E}(|I|) \rightarrow K \tilde{K}
$$

where $K=\mathbf{E}\left(\left|\epsilon_{1}(t)\right|\right)$. Thus, since $\bar{S} d M\left(j_{1}, 0\right)=\mathbf{E}(|I+I I+I I I|)$,

$$
\left|\bar{S} d M\left(j_{1}, 0\right)-\widetilde{K} K\right| \leq|\mathbf{E}(|I+I I|)-\mathbf{E}(|I|)|+\mathbf{E}(|I I I|) .
$$

imsart-aos ver. 2013/03/06 file: multifractals_aos6.tex date: October 22, 2014 
From the Lemma, we know that $\lim _{j_{1} \rightarrow-\infty} \mathbf{E}\left(\left|\eta_{j_{1}}\right|\right)=0$ and consequently $\lim _{j_{1} \rightarrow-\infty} \mathbf{E}\left(\left|\tilde{\eta}_{j_{1}}\right|\right)=0$ which leads to $\lim _{j_{1} \rightarrow-\infty} \mathbf{E}(|I I I|)=0$. Moreover

$$
|\mathbf{E}(|I+I I|)-\mathbf{E}(|I|)| \leq \mathbf{E}(|I I|) \leq \sqrt{\mathbf{E}\left(\left|I I^{2}\right|\right)} .
$$

From the expression of $I I$ and the fact that $\bar{\epsilon}_{2^{j_{1}(\alpha-1)}}\left(2^{-j_{1}} u\right)$ is a $2^{j_{1} \alpha}$-dependent process, we have, when $j_{1} \rightarrow-\infty$ :

$$
\begin{aligned}
\mathbf{E}\left(|I I|^{2}\right) & \leq\|\psi\|_{\infty}^{2} \int_{0}^{1} \int_{0}^{1} \mathbf{E}\left(e^{\omega_{2^{j_{1} \alpha}}^{1}(u)+\omega_{2^{j_{1} \alpha}}^{1}(v)}\right) \mathbf{E}\left(\bar{\epsilon}_{2^{j_{1}(\alpha-1)}}\left(2^{-j_{1}} u\right) \bar{\epsilon}_{2^{j_{1}(\alpha-1)}}\left(2^{-j_{1}} v\right)\right) d u d v \\
& \leq\|\psi\|_{\infty} \mathbf{E}\left(\bar{\epsilon}_{2^{j_{1}(\alpha-1)}}^{2}\right) 2^{j_{1} \alpha} \mathbf{E}\left(e^{2 \omega_{2^{j_{1} \alpha}}^{1}}\right) \simeq 2^{j_{1}(\alpha-F(2)},
\end{aligned}
$$

which goes to 0 provided whe choose $1>\alpha>F(2)$. Thus $\bar{S} d M\left(j_{1}, 0\right)$ converges to $\widetilde{K} K$ which proves (34).

Appendix F: Scaling properties of MRW Scattering Moments

An MRW process is obtained by performing a change of variable in a Brownian motion $B(t)$ with an MRM $M(t)$, as the limit when $l$ goes to 0 of

$$
X_{l}(t)=l^{\frac{2-\zeta(2)}{2}} \int_{0}^{t} e^{\omega_{l}^{L}(u)} d B(u)
$$

where $d B(u)$ is the standard Wiener noise.

Since $B(t)$ is self-similar, one can verify that $X(t)$ inherits the stochastic self-similarity of $M(t)$ and satisfies (26). In particular, one can show (see e.g. [6]) that the multifractal spectrum $\zeta(q)$ of the MRW $X(t)$ defined in (91) is related to the spectrum $\zeta_{M}(q)$ of the MRM $M(t)$ defined in (??) through:

$$
\zeta(q)=\zeta_{M}(q / 2) .
$$

The following theorem gives the MRW version of the Theorem 4.2 for MRM.

Theorem F.1. Let $X(t)$ be a Multifractal Random Walk as defined in (91) with integral scale $2^{L}$ and scaling exponents $\zeta(q)$. Then

$$
\forall j<L, \quad \tilde{S} X(j)=2^{j \frac{3-\zeta(2)}{2}},
$$

and if $\zeta(2)>1$ then as long as $j_{1}, j_{2}<L, \widetilde{S} X\left(j_{1}, j_{2}\right)$ depends only on $j_{1}-j_{2}$, and for each $j_{2}<L$ :

$$
\lim _{j_{1} \rightarrow-\infty} \widetilde{S} X\left(j_{1}, j_{2}\right)=\tilde{K}
$$

where the constant $\tilde{K}$ is the constant of theorem 4.2 for the Multifractal Random Measure associated with $e^{\omega_{l}^{L}}$ in (91).

imsart-aos ver. 2013/03/06 file: multifractals_aos6.tex date: October 22, 2014 
The proof is very similar to the proof of Theorem 4.2 for MRM, so we only provide the main steps without entering into details.

First let us remark that if $\bar{\psi}(t)=\int_{-\infty}^{t} \psi(u) d u$, then a simple integration by parts allows one to show that:

$$
\left|X_{l} \star \psi_{j}\right|=2^{j}\left|d X_{l} \star \bar{\psi}_{j}\right|
$$

where $X_{l}(t)$ is defined in $(91)$ and $d X_{l}(t)=l^{\frac{2-\zeta(2)}{2}} e^{\omega_{l}^{2 L}(t)} d B(t)$. Then, in order to study the behavior of first and second order scattering moments of $X_{l}(t)$, one can adapt the proofs of the MRM case to the MRW case by replacing $e^{\omega_{l}(u)} d u$ by $l^{\frac{2-\zeta(2)}{2}} e^{\omega_{l}^{2}(u)} d B(u)$.

For the first order moment, the Wiener noise scaling $d B(s u) \stackrel{\text { law }}{=} s^{-1 / 2} d B(u)$ and (32), leads to $d X_{s l}(s u) \stackrel{\text { law }}{=} e^{\Omega_{s}} s^{1 / 2-\zeta(2) / 2}$. Thanks to (95), one gets (93).

As far as the second order scattering moment is concerned, a simple adaptation of Lemma D.2 allows one to follow the same steps as in Appendix E. One is lead to the same decomposition as in (86):

$$
\lim _{l \rightarrow 0} \psi \star\left|\psi_{j_{1}} \star X_{l}\right|(0) \stackrel{\operatorname{law}}{=} e^{\Omega_{2}-L}(I+I I+I I I),
$$

where

$$
\begin{aligned}
I & =\tilde{K}^{\prime} 2^{j_{1} \frac{3-\zeta(2)}{2}} \int \psi(-u) e^{\omega_{2^{j_{1} \alpha}}^{1}(u)} d u, \\
I I & =2^{j_{1}} \int \psi(-u) e^{\omega_{2^{j_{1} \alpha}}^{1} \bar{\epsilon}_{2^{j_{1}(\alpha-1)}}\left(2^{-j_{1}} u\right) d u,} \\
I I I & =2^{j_{1}} \int \psi(-u) \tilde{\eta}_{j_{1}}^{\prime}(u) d u,
\end{aligned}
$$

where $\eta_{j}^{\prime}$ is the noise term corresponding to $\eta_{j}$ in Lemma D.2 and

$$
\tilde{K}^{\prime}=\mathbf{E}\left(\left|\lim _{l \rightarrow 0} \int \bar{\psi}(u) l^{\frac{2-\zeta(2)}{2}} e^{\omega_{l}^{1}(u)} d B(u)\right|\right) .
$$

Since from (84),

$$
\mathbf{E}\left(\left|\lim _{j_{1} \rightarrow-\infty} \int \psi(-u) e^{\omega_{2}^{j_{1} \alpha}(u)} d u\right|\right)=\tilde{K}
$$

the term $\mathbf{E}(|I|)$ behaves, when $j_{1} \rightarrow-\infty$ as $\tilde{K}^{\prime} \tilde{K} 2^{\frac{3 j_{1}-\zeta(2)}{2}}$. The contribution of the terms II and III can be shown to be negligible following the same arguments as in Appendix E. Since the first order scattering moment behaves like $\tilde{K}^{\prime} 2^{j_{1} \frac{3-\zeta(2)}{2}}$ we obtain (94) with the same constant as in Theorem 4.2 .

imsart-aos ver. 2013/03/06 file: multifractals_aos6.tex date: October 22, 2014 
Appendix G: Proof of Theorem 5.1

The Fourier transform $\Psi(\omega)$ of $\psi$ satisfies the Littlewood-Paley condition (3)

$$
\sum_{j=-\infty}^{\infty}\left(\left|\Psi\left(2^{j} \omega\right)\right|^{2}+\left|\Psi\left(-2^{j} \omega\right)\right|^{2}\right)=2
$$

It results that

$$
\left|\Phi\left(2^{M} \omega\right)\right|^{2}+\frac{1}{2} \sum_{j=-\infty}^{M}\left(\left|\Psi\left(2^{j} \omega\right)\right|^{2}+\left|\Psi\left(-2^{j} \omega\right)\right|^{2}\right) \leq 1 .
$$

Let $Y$ be a stationary process with $E\left(|Y(t)|^{2}\right)<\infty$, and $P_{Y}(\omega)$ be its power spectrum. Multiplying (100) by $P_{Y}(\omega)$ and integrating in $\omega$ gives

$$
\mathbf{E}\left(\left|Y \star \phi_{M}\right|^{2}\right)+\sum_{j=-\infty}^{M} \mathbf{E}\left(\left|Y \star \psi_{j}\right|^{2}\right) \leq \mathbf{E}\left(|Y|^{2}\right) .
$$

Let us prove by induction that for any $q \geq 2$

$$
\begin{aligned}
& \mathbf{E}\left(\left|\widehat{S} X\left(j_{1}\right)\right|^{2}\right)+\sum_{m=2}^{q-1} \sum_{-\infty<j_{2}, \ldots, j_{m} \leq M} \mathbf{E}\left(\left|\widehat{S} X\left(j_{1}, \ldots, j_{m}\right)\right|^{2}\right)+ \\
& \sum_{-\infty<j_{2}, \ldots, j_{q} \leq M} \mathbf{E}\left(\left.|| X \star \psi_{j_{1}}|\star \ldots| \star \psi_{j_{q}}\right|^{2}\right) \leq \mathbf{E}\left(\left|X \star \psi_{j_{1}}\right|^{2}\right) .
\end{aligned}
$$

Applying (101) to $Y=\left|X \star \psi_{j_{1}}\right|$ proves that (102) for $q=2$. If (102) is valid for $q$ we prove it for $q+1$ by applying (101) to each $Y=|| X \star \psi_{j_{1}}|\star \ldots| \star \psi_{j_{q}} \mid$. Taking the limit of (101) as $q$ goes to $\infty$ proves (102).

If $\psi$ has a compact support then one can verify that there exists $\phi$ having the same support as $\psi$, whose Fourier transform satisfies (36), with an equality on the Fourier transform modulus. Since $\mathbf{E}\left(\widehat{S} X\left(j_{1}, \ldots, j_{m}\right)\right)=$ $\bar{S} X\left(j_{1}, \ldots, j_{m}\right)$, the mean-square estimation error at the scale $2^{j_{1}}$ satisfies

$$
\epsilon\left(j_{1}\right) \leq \sigma^{2}\left(\left|X \star \psi_{j_{1}}\right|\right)-\sum_{m=2}^{\infty} \sum_{-\infty<j_{2}, \ldots, j_{m} \leq M}\left|\bar{S} X\left(j_{1}, \ldots, j_{m}\right)\right|^{2},
$$

which proves (37) $\square$. 